\title{
Patient Outcomes Registry
}

National Cancer Institute

\section{Source}

National Cancer Institute. Patient Outcomes Registry. NCI Thesaurus. Code C119669.

A registry that uses observational study methods to collect uniform data to evaluate specified outcomes for a defined patient population, and that serves a scientific, clinical, or policy purpose. 